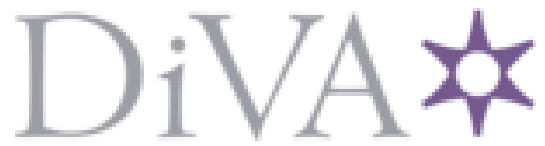

http://www.diva-portal.org

Preprint

This is the submitted version of a paper presented at FIE 2018, October 3-6, San Jose, CA.

Citation for the original published paper:

Grande, V., Peters, A-K., Daniels, M., Tedre, M. (2018)

"Participating under the influence": How role models affect the computing discipline, profession, and student population

In: Proc. 48th ASEE/IEEE Frontiers in Education Conference Piscataway, NJ: IEEE Press

https://doi.org/10.1109/FIE.2018.8658944

N.B. When citing this work, cite the original published paper.

Permanent link to this version:

http://urn.kb.se/resolve?urn=urn:nbn:se:uu:diva-364748 


\section{"Participating Under the Influence": How Role Models Affect the Computing Discipline, Profession, and Student Population}

\author{
Virginia Grande, Anne-Kathrin Peters, Mats Daniels \\ Uppsala University \\ \{virginia.grande, anne.peters, mats.daniels@it.uu.se\}@it.uu.se
}

\author{
Matti Tedre \\ University of Eastern Finland \\ matti.tedre@acm.org
}

\begin{abstract}
This full paper in the research track presents how individuals in computing education may have role models that represent different ways of engaging in the discipline and/or profession as a student or a professional. The study is based on two rounds of interview-based data collection at a department of computing: a longitudinal study of undergraduate students' view of the discipline, and an examination of their teachers' experiences as role models in computing education. Our results challenge traditional views of role models as those who set the norms, presenting instead role models as potentially capable of change, at different scales (including none), depending on their level of power. These role models are students, academics, and other professionals. We show that the potential of role models must be understood with respect to how engagement in computing is constructed in social interaction. Actions are suggested for relevant stakeholders to reflect on which role models are receiving more or less exposure than they should and how through these role models participation in computing can be broadened in terms of not only diverse backgrounds but also ways of engaging in computing.
\end{abstract}

Keywords-role models; computing; identity; discipline; profession, computing education

\section{INTRODUCTION}

In the Western world, underrepresentation and lack of inclusion of certain demographics in computing are a continuous political and economic concern [1], [2], [3], [4]. There is a long history of research trying to address these issues [5]. However, the concerns remain. Recent STEM education research points out that these problems have been investigated mostly with a focus on understanding learners' deficits in interests, motivation, and competence, with the aim to fix those deficits in educational initiatives [6], [7]. These researchers suggest that efforts towards these ambitions could benefit from a wider understanding of learners in interaction with the learning environment. We build on this approach, especially taking the influence of role models into account.

Role models are often part of strategies for recruitment and retention of students [8], [9], [10] (particularly from underrepresented groups [11], [12], [13]) because they offer a concrete representation of a particular skill or trait that can be imitated by a student. This has been studied even for small children [14]. Some benefits of having a suitable role model are positive effects on a student's motivation [15] and on academic achievement [16].

In this paper we develop the concept of role model further, especially in relation to understanding role models as part of a social context in which what it means to engage in the discipline and profession is negotiated, which affects and constrains how individuals can develop as computing persons. Social identity theory has been suggested as a "link" to understand the interplay between social structure and individual development [17]. It is used as a theoretical foundation to advance our understanding of the phenomenon of role models and how role models can be used to broaden participation in computing.

There are many ways of engaging in computing. These different ways can be made concrete by the examples given by role models. Students may see in their role models the reflections of disciplinary and professional identities that they aspire to have for themselves. However, in our work we have seen that in reality only a subset of these reflections are actually available as role models to computing students. While one of the expected positive outcomes of role modeling is broader participation, we give examples that show how this is very complex. For example, the design of study programs and curriculum, or who holds leadership positions (that is, who the community seems to consider as important) affect who the students may perceive as more influential or who influences the students in crucial stages of their development as computing persons.

To establish a new understanding of role models, we draw on two empirical studies at Uppsala University: a longitudinal study that followed computing students through their education and an investigation of teachers' experiences of role modeling. We connect these studies to existing research on the history of computing and computing as a profession.

We use these studies and examples from history to encourage reflection on the varied levels of influence of role models for students, with these role models being not only teachers but also students. We argue for the need to study role modeling as a cultural complex phenomenon that needs to be addressed by stakeholders and policy-makers with the power to change the culture of computing at different levels.

\section{ROLE MODELS}

The use of the term role model varies in the literature and even in everyday life. This is because the term is loosely defined and thus used in different ways [18], [19]. While most definitions seem to have in common a core concept, i.e. a role model being someone to imitate, they differ in the details. Looking at different dictionaries, one encounters that some highlight how a role model may tend to be older 
than the person imitating them [20], others emphasize the admiration for the model [21] and others specify that the imitation is limited to the particular role presented by that role model [22]. These differences are important because they have already affected study results when trying to analyze role modeling [23]. While the definition is assumed to be universal, a researcher may provide their study participants with a common definition. Despite that measure, participants still give their own meaning to the concept and thus their answers may varied due to their different interpretations [23]. For example, a student may admire their teacher but not want to become them, so they do not refer to this educator as a role model [23]. Or one may agree that a role model is someone outstanding and worthy of admiration but age may not be considered part of the equation, while some other participant may not even stop to consider a younger person as a role model due to their perceived lack of experience.

Similar discrepancies occur in the academic world. Here we present several definitions and how they differ. When referring to the individual emulating the role model, we use emulator for brevity. For Haveman and Wolfe, role models are "adults or peers to whom children or adolescents relate and who set norms of behavior and achievement to which they aspire [24]". Gibson's [18] definition of role modeling focuses instead on the emulator having a goal and using role models as a means to get closer to achieving that goal, through the emulation or avoidance of certain aspects of the model's attributes or behaviors. Both lines of work include a connection between role model and emulator: the emulator needs to perceive that they are similar -to some extent- to the role model. While Haveman and Wolfe see role models as individuals with a certain level of power (at least, to set norms), Gibson portrays role models simply as possible exemplars of the professional competencies and personal attributes that the emulator may need to achieve a goal [18]. This exemplification is an embodiment of certain traits or skills, that is, the role model makes concrete an idea that was abstract so that the emulator can choose to copy it [25].

There is yet another difference between these definitions. Most popular and academic definitions focus on role models as examples to follow for a desirable outcome. However, Gibson's definition includes a reference to avoiding the emulation of the aspects of the role model. This is because Lockwood [26] divides role models in two kinds: positive and negative. A positive role model refers to the common interpretation of role models as individuals to imitate, while negative role models represent aspects to avoid in order to achieve goals. For example, to follow a healthy lifestyle, one may choose to imitate an athlete regarding habits to exercise (positive role model) and to avoid copying the smoking habit of a smoker (negative role model).

That an individual considers another person as a role model depends on whether the model's success seems attainable [27]. If this is so, role models motivate emulators to achieve greater goals. The level of motivation depends on how positively the individual regards themselves [15]. As goals change according to career stage, career stage affects the choice of role models: Gibson talks about early, middle and late career differences [18]. Culture can be another factor: emulators from collectivist cultures tend to look for negative role models, while in individualistic cultures the tendency is to search for positive role models [26].

In terms of connection with the role model, the emulator's perceived similarity to the role model is significantly related to self-esteem, particularly for women [28]. In an educational context, when the gender and ethnicity of a role model for students match with the students', these students' academic achievement is higher than that of students with no match in this regard or no role models at all [16]. Women rate their role models as more important to them than men do [29]. When female students have contact with female STEM experts, they value these role models higher than men do [30], while for men the gender of their role model does not determine the impact of this model [31].

In this paper we incorporate the above definitions to our findings, resulting into the following definition: A role model in computing is an individual who embodies one or more desirable ways of engaging with the discipline and/or profession. Both the role model and the emulator can be a professional or a student, in any combination. Whether an individual emulates a role model depends on the social context, particularly on the level of influence that the role model has. We unpack this definition in the following sections.

\section{IDENTITY}

Jackson and Pozzer [32] identify two conceptions of identity in STEM education research: identity as something that is possessed by an individual, and identity as something that is negotiated in interaction. In the first approach, identity is studied as something similar to a personal trait or characteristic. In the negotiation approach, identity is viewed as something outside the individual, something that is constructed in social interaction, which affects and is affected by individuals. The negotiation approach to identity is interesting for the purpose of this paper to understand role models as part of social contexts. We reason about the role modelling of people who conform and do not conform to social norms.

The following three concepts are central in the identity literature [33]: power, agency, and uniformity. Power relations constrain becoming, but they can also be seen as "the very condition of" an individual" existence [34, p. 2]. A role model could therefore be seen as a person that stands in a power relation to another person as it shapes that person. Agency is used as a term to reason about the extent to which people can be agents of their becoming and their behaviour. There are theoretical frameworks that argue that people have little agency, that people are formed by social interaction and language (see e.g. [35]). With such an assumption, following role models cannot be thought of as an individual process. The notion of uniformity can be used to reason about whether or not individuals can be understood as an integral, unified identity. A person as uniform has been criticized in a variety 
of disciplinary areas [36, p. 1]. We can therefore consider that a person may be able to emulate a role model in one (allowing) context, but not in another (limiting) context.

\section{DisciplinARY AND PROFESSIONAL IDENTITIES}

Starting from the most basic levels, disciplinary identities can involve structural elements (home departments, buildings, memberships in organizations), and cultural elements (values, beliefs, norms, ethics, traditions, heroes) [37]. Disciplinary identities can involve one's views of the field's problem-solving practices, methods, and tools. Another window at the constituents of a disciplinary identity is to view it in terms of a shared paradigm or a research agenda: a consensus between practitioners of a discipline over the kinds of problems the discipline deals with, their priorities and importance (what is hard, what is easy), how those problems should be solved, what theories on which fields one can rely on when solving them, and how solutions to those problems look like [38], [39, p 130].

Modern views of disciplinary (and professional) identities follow the postmodernist tradition in the sense that individuals continuously construct and re-construct their academic and professional identities, values, attitudes, knowledge, and practices, and that there is no single identity but many intersecting identities [37]. Like other theories of identity, also disciplinary identities form and manifest in a multiplicity of ways; for instance, through divisions between in-group and out-group; through boundaries (of one's authority, autonomy, or expertise, for instance); and through specialist vocabulary, rhetorics, and discourse [40]. In education, disciplinary identity and disciplinary ways of thinking and practicing have been highlighted in, for instance, studies of threshold concepts and troublesome knowledge [41]. From the birth of computing as a discipline, the field's academic identity has been characterized by multiple "cultures" [42] or "traditions" [43] of computing. Traditions manifest at multiple, crosscutting levels of abstractions, in form of intellectual traditions (e.g., theoretical, empirical, and engineering), research traditions (e.g., analytical, design, and experimental), and methodological traditions (e.g., formal methods, case study research, and contextual inquiry), to mention a few. Debates between the merits of each tradition have sometimes been fiery: For instance, in the 1970s and 1980s the proponents of formal verification and software engineering clashed over whose methods would better improve trustworthiness of computer systems.

The theoretical tradition saw computing primarily as a study of formal, mathematical objects, such as algorithms, complexity classes, and computational models. One does not need a computer to study formal objects: they can be studied with a pen and paper as well [44]. Correct results about them are necessarily true within a system of formal rules. The empirical tradition saw computing primarily as a study of empirical objects, such as computer systems, executable programs, and live computations [44]. Studying them requires building them, setting up experiments, and evaluating the results. The design tradition saw computing as a study, design, and creation of artifacts, such as user interfaces, software, and hardware. Artifacts are human-made objects based on some needs or desires, and their design requires understanding of material, theoretical, and sociotechnical aspects of their intended use.

One's disciplinary identity was affected by the schools of thought one belonged to: A proponent of formal verificationism saw the field as a chiefly mathematical field that uses the formal tools of mathematics and aims at proofs of correctness of computer systems, while a software engineer saw the field as a primarily design-oriented field that relies on engineering heuristics and patterns, and aims at building reliable, usable, and safe systems [45]. The different schools of thought in computing have always given rise to a broad spectrum of disciplinary identities, each of which has its own sets of problems, practices, methods, tools, exemplars, aims, and priorities [46]. They often also have their own idols, organizations, values, vocabularies, rhetorics, and discourses.

Different facets of computing's multi-dimensional identities are visible in the field's many specializations. Designand engineering-oriented identities are prevalent under labels like programmer, software engineer, and user experience designer. Empirically oriented identities can easily be found under labels like data scientist, computational physicist, and systems analyst. Theoretically oriented identities are often found with those who work on, for example, formal verification, complexity theory, and cryptography. However, as computing's traditions are deeply intertwined and very rarely appear alone, computing's disciplinary identities are also multifaceted and complex [46].

Professional identity has been theorised by Nyström [47], where she points out that professional identity is negotiated relationships between professional, personal, and private life spheres. Work by Hodkinson et al. [48] indicate a discrepancy between professional identity formation in higher education and in working life regarding what knowledge is prioritized and how to practice the profession. The term disciplinary identity is in this paper used to refer to how we tell each other who we are in relation to the theoretical and technical core of the discipline, which is prominent in higher education contexts. The term professional identity is used to also include aspects related to using competence in the disciplinary core in complex work and social contexts.

Research suggests that computing is constructed as doing the technical in professional environments. Faulkner [49] e.g. finds two identities, a technical engineering identity and a more heterogeneous identity, whereby engineers seem to be drawn to a technical identity. She argues that masculinity and the profession are co-produced as engaging in the technical.

\section{IDENTITIES In Computing EdUCATION AND STUDENT DEVELOPMENT}

A longitudinal study [33] that has been conducted with the aim to explore students' relationship to the discipline and how it changes as the students engage in their studies, suggests that certain disciplinary identities are accessible in education while others are not. Students of two computing 
study programs at a Swedish University, Computer Science (CS) and Computer and Information Engineering (IT), were followed over a three-year period. All students that commenced the programs in 2012 wrote essays about their choice of study, career aspirations, and study expectations early in their education, upon which 23 students were selected to follow through interviews. Group interviews were conducted to discuss the results of the study with the students.

While the participants of the longitudinal study reflected on various ideas about computing when they entered their study program, they experienced computing in rather narrow and technical ways later on in their studies. Students who commenced the study program with broader interests in society, art, or helping people, dropped these ideas, some of them struggled to make sense of their studies or changed to a different study program that the students perceived to be more relevant for their broader interests.

The focus of the analysis became how the students experience participation in their field of study [33], doing, thinking, and feeling in relation to the field of study, as something that is negotiated in interaction among different people. The analysis suggests that there are two predominant ways of participating in computing: participation as creating digital artifacts and technical problem solving. Participation as creating seems to be the focus among students. When the students sit together in their study breaks, they commonly discuss ideas for apps and games to develop. Being able to implement these ideas and develop small programs is experienced as fun. Participation as problem solving includes creating programs, but it is also about solving difficult technical problems that appear when developing digital artifacts. Difficult problems are divided into smaller problems that are solved separately. Solving different types of subproblems is central in the education. The broader application context seems less relevant, participation as problem solving is experienced as something technical.

In the third study year, the students took a course in human computer interaction (HCI) that was rejected by many participants of the longitudinal study as out of the scope of computing. The course covers aspects such as usability, requirement elicitation, and inclusive design that are necessary in the context of the engineering-design tradition of computing. Aspects of the HCI course should be relevant to students with an interest in learning to create digital artifacts, and for students that are concerned about making a difference for people or society. However, the students were hesitant about the course or dismissed it.

These findings are in-line with previous research that suggests that computing is constructed in ways that center around the computer, programming or coding [50], [51]. Computing curricula focus on technical aspects of programming and mathematics in early years, deferring multidisciplinary projects to the end [51], [52]. Cech [53] describes a culture of disengagement with public welfare in engineering and finds that the students' public welfare concerns decline as they proceed in their studies.

The data includes several examples that suggest that it is not legitimate to show broader interests, thus to perform a more heterogeneous identity. The following quote e.g. shows the reactions and interactions that someone performing in such broader ways can invoke:

Chris ${ }^{1}(\mathrm{CS}$, year 3): The teacher [of the HCI course] was very interested in HCI. [...] We thought: 'He is not a real computer scientist!'. (laughs) But then it turned out that he actually could program and that he was as good as we are, $[\ldots]$ just that he had an interest for that which was a bit fuzzy.

Social constructions of computing need to be understood in order to understand who the students become as computing people and which role models they emulate. Several reports explain engagement and diversity issues with narrow student perceptions of the discipline. Initiatives to address students' lack of knowledge and interests are advocated (see e.g. [1]). Role models are installed to make missing aspects visible. However, the example of the HCI course shows the complexity and challenges involved aiming for increased diversity. While the teacher of the HCI course could be a role model for the students because he engages in different ways than the students were used to, the students' reactions, questioning $\mathrm{HCI}$ as an area of computing, prevented, or at least limited, the teacher's power as a role model. Perhaps the teacher being openly rejected by the students even reinforced a culture that excludes a design-engineering identity or being someone interested in less technical aspects.

\section{TEACHERS AS ROLE MODELS}

The literature on role models focuses on the perspective of the emulator. There seems to be a lack of research on the perspective of the role models themselves. In particular, there is a gap to fill when the teaching context is considered: when teachers may act as role models for their students. Thus, we conducted a phenomenographic study of teachers' experiences of role modeling for their students at the Department of Information Technology of Uppsala University.

For an interview study we selected as participants 9 teachers with different backgrounds, pedagogical experience, area of research expertise, gender, and age. We recorded and transcribed the interviews. The teachers were asked about how they experienced potentially being a role model for their students and about other role models for their students that the teacher may make part of their teaching [25].

Results show that a teacher may consider what they, as a role model, embody (personal or professional qualities, positive or negative), how this can be perceived by others (by students of different groups and colleagues), and who it may affect (students and society). The participating teachers talked about how role modeling means knowing the subject domain and transmitting interest for it. Here, even if not explicitly, they were referring to teachers as examples of disciplinary identities. Role modeling for these teachers also meant displaying personal traits and skills related to the

\footnotetext{
${ }^{1}$ All names are not real names.
} 
profession. For example, they want to show students how to treat everyone fairly or presentation skills.

Sometimes the teacher's embodiment of the skills could be, they thought, considered as subpar or even flawed behavior. Thus, there was a reflection on how role modeling in computing education is not only about what the teacher intends to transmit but also about how this is perceived by the student, regardless of the teacher's intention.

It was mentioned that a teacher's role modeling may not be applicable to all students but only (or more predominantly) to a subset of the cohort. This depended on the perceived similarities between emulator and role model and, sometimes, on the goals of the emulator, as one interviewee pointed out:

Robin $^{2}$ : [...] having a teacher as a role model, then it could be more about [imitating personal traits].[...] [the skills modeled by a teacher] are related to the teaching and the student might not necessarily want to be a teacher.

Robin seemed to believe that a teacher can be an example of disciplinary identities but not necessarily of professional identities (other than as a teacher).

While role modeling tends to be described as the impact of one model on another individual, some teachers looked at the wider context and considered how a teacher's role modeling may affect people other than their students. Daniel talks about both the development of discipline and the responsibility to provide students with the skills to be professionals in the field:

Daniel: [...] the conflict about what do we want, I mean, what do we want to achieve with the teaching. [...] I want to actually further the subject. [...] Most of [the students] will end up out in society in some kind of jobs and of course it is my duty to, mmmm, make sure that they have the necessary skills to do that effectively.

This responsibility towards society is also mentioned by another teacher, Joe:

Joe: We are training academics, the industry leaders of the future and... yeah... I guess [the students] are going to look at the behavior and say "I guess this is acceptable behavior" and if we behave poorly, [...] could be an example of poor behavior that can.... yeah. Can have a very negative influence...

Joe emphasizes that teachers can have an impact on their students as role models for them. As these students become academics and professionals in industry, they affect society as "leaders of the future". This effect on society includes their teacher's influence as a role model. More importantly, this influence is not necessarily always a positive one. If a teacher embodies poor behavior that is copied in the future by academics and professionals in industry, work environments and the influence of the field in society can take a negative turn. Conversely, a teacher's positive behavior may have a larger impact than just on their students.

\footnotetext{
${ }^{2}$ All teacher names are not the teachers' real names.
}

\section{Role Models' VARIEd Power}

Students look to their peers for examples of how to engage in computing. One of the salient examples is - in the words of the longitudinal study participants - the "skilled" student that others perceive as knowledgeable or good at a particular subject. Skilled seems to be mostly seen in terms of being good as programming. Kim, for example, reasons:

Kim (year 2, IT): There are many students who only see their education as an opportunity to become better at programming, it is the only thing they want to do and they are really good at it. Whereas I see IT as a springboard to something else, to something where I can sit and help other people instead of programming all day long.

Apparently there is a large student group who shares a strong interest in programming without thinking much about contributing or helping others. Kim sees himself as different. He talks about avoiding behaving like these other students (who, in that way, are acting as negative role models). In the interview situation, he seems to feel safe to distance himself from those students who follow the norm. This may not be the case in social contexts of the learning environment (see uniformity, Section III).

The students that other students observe are not only from their same year of studies but also from the following years. Amari, another participant in the longitudinal study, says in an interview in year 3 that he is very interested in algorithms. He tells about the algorithm and data structure class that was about learning many different algorithms, and also developing and implementing algorithms to solve different problems. Amari says that he wishes he would have had more time to read to learn more than what he did listening to the lectures.

Amari (CS, year 3): We have this bible, [...] a thick book which contains a lot of algorithms [...] and data structures. [...] it is very complete and [...] it contains only necessary text. [...]

Interviewer: How did the book get the name bible? Amari: [...] Older students have called the book 'the bible', because for many students, CS is all about algorithms and data structures.

This quote suggests that older students can affect Amaris' interests and engagement in computer science. They define what it means to engage in computer science and do not leave much room for other role models.

But do students always have the power to influence their peers? A student in the IT in Society course at Uppsala University [54] tried to convince the others in the project team to take ethical aspects seriously. He had little success. But everything changed when a company representative, who was seen as a technical expert, conveyed a strong interest in the issues the student tried to raise. It was only then that the group followed the student's suggestions. This example illustrates how a student may have little to no influence on peers without the support of other, more influential actors. The source of this power of influence could be the kind of identity 
that the role model represents, for example a technical-related identity. It also is another example of students dismissing a way of engaging in computing that includes considering how their work affects people. The other students regarded their peer's comments in this area as irrelevant and, more importantly, complicating the solution the team wanted to develop. The shift when the whole project changed to clearly address the more open and complex aspects raised by the student came from a genuine shift in attitude towards the importance of being able to connect technology issues to a wider societal context. The incoming (powerful) role model changed the educational context in a way that allowed the student to become a role model possible to emulate.

Professionals who are regarded as experienced are also role models, individuals from which to learn acceptable behavior particularly for those in a new environment. Joe, a teacher in our study, reflects on how he thought that some academics transmit questionable attitudes:

Joe: we [teachers] are all putting ourselves out there in our behaviors... observed and judged and.... by the students... adapted or adopted whether we behave poorly or... otherwise, well, I remember, this was a student who started, he remembered sitting in meetings discussing research where people were being really aggressive and he thought "OK, this is the way I have to be" $[. .].[$ T] hey were role models whether they wanted to be it or not, they were bad examples of how you should behave.

Here Joe mentions researchers who are, from his perspective, negative role models (examples to avoid). Notice, however, that for the student they are seen as positive role models (examples to follow) according to Joe's perception. They are shaping the student's view of what it means to engage in research in computing. As described in section VI, Joe also reflected on how this shaping affects not only this particular student but also the field as a whole when the students become professionals in academia or industry.

This concern regarding the effect of role models to disciplinary formation is supported by examples from history, such as that of formal verification in the 1970s-1980s [55]. There were a group of very vocal people who believed that the best way to build reliable systems is by formally verifying them. They were able to gather a following of ardent supporters of that view [55]. Historians of computing have shown how following those role models had ramifications that affected whole national research agendas. For instance, a few strong role models dominated Dutch computer science, which had implications on the Dutch computing scene for decades.

Some aim to be role models for what they consider a positive change of not just the discipline and profession, but of how certain members of the computing community are expected to behave in this and other communities. Robin, a woman teaching in a male-dominated area, talks about deliberately showing students of all genders attitudes that go against gender-related norms, such as not always being as likable as possible in several aspects:
Robin: saying no to things to not perpetuate stereotypes [...], I don't want [the students] to copy that [behavior stereotypically attributed to women]. [...] I try to be less friendly and smile less because I don't think I should be friendly with everybody just because I'm a woman and we tend to do that, apparently.

However, not all role models succeed, completely or even partially, in having an impact on their emulators. We have seen that some role models have enough power to have an effect, while others do not (see example in section $\mathrm{V}$ where the HCI teacher is not seen as a positive role model). While what it is desirable to emulate (and, thus, who a role model is) is subjective and depends on each individual, we have shown that this is highly influenced by the social context of the potential emulator. Particularly, how the community values the potential role model, as this evaluation is perceived by the emulator, greatly affects the impact of the role model. In fact, it can determine whether that individual is considered as an example worth imitating.

\section{DISCUSSION}

We identified three types of role models according to what they represent: disciplinary identities, professional identities, and ways of being students in computing. The same person can act as more than one of these categories, as we illustrate for both students and teachers. However, not all of them have the same level of influence on their emulators or of endorsement by the community.

A student may act as a role model for a peer in more than one way: for example, as a role model of how to behave as a professional, e.g. being perceived as a very good programmer, and also of how to pass a course. This challenges the popular view that a role model is someone significantly older than the emulator. Students that are acting as role models seem to be perceived as more experienced by the emulator though, regardless of their actual experience.

Our studies provide insights into the kind of role models available for students. The interviews with the teachers suggest that teachers' intention is to embody both professional and disciplinary identities. Students, on the other hand, have a strong focus on technical elements of the discipline.

There are disciplinary and professional identities that need to be represented in a stronger way in the educational system. This can be done through role models. In this sense, role models would help broadening participation not only in all forms of diversity (e.g. gender, ethnicity) but also in terms of engaging in computing as a discipline, profession, and study degree. Students need to see examples of people in computing that consider ethical aspects and the user, sustainable development, and others.

However, working towards this goal is complex. There are many factors, which are in turn also complex. For example, we have shown that the power of influence the role model has is something very relevant to consider. But where this power comes from has no trivial answer. 
Consider for instance how the hierarchy in education (the distance between teachers and students) varies depending on the culture of the country and, as a result, so does the level of influence of a teacher. This is not the only variation in perception of teachers. Macnell et al. conducted one of the experiments that show how students rate male teachers higher than they rate female teachers due to gender bias [56]. This kind of bias is also present in the opposite direction in the relationship between the teacher and students: a study simulating the hiring process of a student for a lab managing position showed that teachers of all genders perceive male students as more competent than their female counterparts, and offer them more opportunities of mentorship and a higher salary [57]. These biases affect whether an individual (teacher or student) is perceived as a role model. The previous study suggest that women are less likely to see as competent, and thus, as role models. This is especially problematic, considering that women are value their female role models higher, as mentioned in section II. Biases also are an important factor to consider when we analyze how likely someone is to present themselves as a role model. While there may be people in the educational system who could be role models, self-applied biases may hinder the person to present their competences and experiences. Women are negatively stereotyped when it comes to math or technical skills. This can affect their view of their own competences and their actual performance [58].

How a person decides to have someone as a role model is in itself also very complex. Gibson states that role models represent an aspect to copy in order to achieve one of the emulator's goals. Those may, however, be very different depending on the individual, considering that the computing community is diverse. Individual goals are dynamic, that means they change over time and social context. The strategies to achieve these goals vary too. For example, we have mentioned how some cultures tend to favor identifying positive role models while others will look for their negative examples to avoid emulating [26].

We have mentioned a few aspects that characterize the complexity of the problem. We have not discussed external factors that affect role modeling, such as society, and globalization. There are also other circumstances that add to the difficulty of this problem, such as the availability of teachers, and ideas that are spread in a learning environment that can be opposed to learning goals.

While there is no easy solution for the lack of representation of certain disciplinary and professional identities, there are actions that we as a community can take. We can study and change the different degrees of exposure of professional and disciplinary identities to emulators through role models endorsed by the community.

\section{CAll FOR ACTiON}

We recommend some reflection points for different actors related to education: those involved in study program design and development, and professional organizations.

\section{A. Study Program Coordinators and Related Stakeholders}

Role models representing diverse ways of engaging in computing can have an impact on students, with this level of influence varying depending on when a course is taught. If courses that emphasize social aspects are not included in the earlier years of a study program, by the time the students take these courses their experience of the discipline has already been shaped too narrowly. In turn, the students' goals are also narrower than they could have been should they have had access to more diverse identities. Potential role models that represent broader ways of participating in computing see, then, their level of influence affected. When designing a study program, it is worth considering which areas of computing are getting less visibility in the program than they should and whether some content should be moved to earlier/later in the program. In the learning environment that we investigated (see section V), the HCI course was moved from the third year of the study program to the second, as a reaction to the results of the longitudinal study.

There can be teachers that are seen as a negative role model by students. For example, students may avoid specializing in an area of computing partly because they want to avoid becoming the teacher that taught a course in that area. Study program coordinators can pay attention to identifying such cases, and act.

Aiming to broaden the accepted ways of engaging in computing, students need to have access to teachers with diverse backgrounds. All experiences beyond the technical are worth sharing, yet currently they are not as visible in computing education. The diversity in teachers' backgrounds can be made more explicit to their students. For example, there are teachers that do not have a background in computing but rather in other areas (for example, those who have degrees in teaching, psychology, or a field natural sciences). Yet the students may be unaware of this diversity in background and assume more heterogeneity among their teachers' education by expecting all teaching staff to have a computer science background. Institutional online profiles, or other platforms where students can check information about their teachers, could be a good starting point for change besides the classroom. Teachers should be encouraged by their leaders to present broad experiences (e.g. experiences from study programs outside computing).

Similar reasoning applies to research. Teachers can talk about their research experience in ways beyond the technical. They can more explicitly highlight in their teaching the multidisciplinary character of their (or others') work. Ethics and sustainability in computing could be topics that extend the technical, that are of concern today. Again, access to all of this information should not come too late.

We have mentioned that the emulator needs to perceive the success of the role model as attainable in order for the role modeling to work. Teachers can reflect on how to share with their students what factors they think have affected their professional success and which of the skills and attitudes related to these factors the students can aspire to learn. In 
particular, teachers can share which factors for their success the students can emulate from the teachers themselves or other role models.

\section{B. Professional organizations}

Professional organizations have a strong influence in the discipline through, for example, their development of curriculum, organization of scientific events, and venues for publication. People who are visible, in different degrees, thanks to professional organizations can be role models for members of the computing education community. For example, in gatherings such as conferences, attendees gain access to keynote speakers, members of the organizing committee, participants who are further in their career, etc. When an emulator perceives similarities with these individuals who have achieved one or more of the emulator's goals, these people can become role models. It is important to analyze, then, what kind of role models are available for what audience at research gatherings. This has been done for underrepresented groups [59]. The same reasoning applies for the leadership, at different levels, of these organizations. Individuals in important positions are regarded as important people in the community, i.e. they may be seen as a reflection of what is valued in the community.

The way these role models are presented to the students (and professionals) needs to be carefully considered. As stated above, for role models to be effective, their achievements need to be perceived as attainable. When choosing speakers and leaders, members of committees can reflect on the following aspects: Are the potential role models communicating both desirable and achievable goals? If so, are they conveying what skills and personal attributes are needed to achieve these goals? Is the organization fostering a connection between emulator and role model, facilitating the perception of similarities between the two of them? Which groups are getting more exposure and which should have theirs increased? Is this exposure leveled within the group? For example, there may be women as role models but how many are in the higher leadership positions? Similar suggestions as those presented in part A of this call for action can be considered.

Editors can consider whether they are giving more visibility to only a subset of areas within computing and whether they can broaden the range of ways of engaging in computing that their associated venues for publication include. This reasoning applies to program committees as well. Overall, professional organizations can reflect on what ways of engaging in computing are represented in their events and venues for publication, and how explicit they are about the diversity in their members' background, multidisciplinary work, and professional and disciplinary identities.

\section{Conclusions}

Individuals in computing education may have role models representing disciplinary identities, professional identities, how to be a student, or a combination of these. We challenge traditional views of a role model as someone who sets the norms, presenting instead role models as potentially capable of change, at different scales (including none), depending on their level of power. Students, researchers, and other professionals can act as emulators and/or role models.

The potential of role models must be understood with respect to the disciplinary culture at the learning institution, i.e. to how engagement in computing is constructed in social interaction. There needs to be reflection on which ways to be in the field are endorsed by our community through role models: who is getting more visibility and who should have theirs increased. Powerful role models not only contribute to broadening the variety of disciplinary identities available to students. They are also an important addition to another toolbox. They can contribute to raising motivation to develop a set of professional competencies in order to become a professional suitable for today's and tomorrow's society.

We may not choose who our students' role models are but we can choose who we (as a community, and individually as teachers) endorse, and support, as role models. As educators and professionals in computing, we have to reflect on what kind of culture we are contributing to through our endorsement (or lack thereof) of role models from different groups and represent different ways of participating in computing.

As the topic of power of role models is complex, there is abundant future work in the area. Here we have only scratched the surface of the professional identities subject. Analysis of other factors related to role modeling and context can be developed, e.g. the impact of gender in role modeling in the learning environment and its intersection with different ways of engaging in computing. Another addition would be identifying who the students report seeing as role models and which group these role models belong to, e.g. role models related to the profession, discipline, the groups to which the students belong. The hierarchy of role models depending on their level of power could be studied in order to understand the different levels of influence.

\section{ACKNOWLEDGMENT}

We are particularly grateful to one of our reviewers, who included so many useful recommendations and insights!

\section{REFERENCES}

[1] The ACM/IEEE-CS Joint Task Force on Computing Curricula, Computer Science Curricula 2013: Curriculum Guidelines for Undergraduate Degree Programs in Computer Science. ACM, 2013.

[2] D. Lövheim, "Scientists, Engineers and the Society of Free Choice: Enrollment as Policy and Practice in Swedish Science and Technology Education 1960-1990," Science and Education, vol. 23, no. 9, pp. 1763-1784, 2014.

[3] E. Smith and S. Gorard, "Is there a shortage of scientists? A re-analysis of supply for the UK," British Journal of Educational Studies, vol. 59, no. 2, pp. 159-177, 2011.

[4] M. S. Teitelbaum, "Do We Need More Scientists?," The Public Interest, no. 153, pp. 40-53, 2003.

[5] S. Beyer, "Why are women underrepresented in Computer Science? Gender differences in stereotypes, self- efficacy, values, and interests and predictors of future CS course-taking and grades," Computer Science Education, vol. 24, no. 2-3, pp. 153-192, 2014.

[6] L. Ulriksen, L. M. Madsen, and H. T. Holmegaard, "What do we know about explanations for drop out/opt out among young people from STM higher education programmes?," Studies in Science Education, vol. 46, no. 2, pp. 209-244, 2010. 
[7] V. Tinto, "Research and Practice of Student Retention: What Next?" Journal of College Student Retention, vol. 8, no. 1, pp. 1-19, 2006.

[8] G. C. Townsend, "People who make a difference: mentors and role models," ACM SIGCSE Bulletin, vol. 34, no. 2, pp. 57-61, 2002.

[9] D. Clayton and T. Lynch, "Ten years of strategies to increase participation of women in computing programs: The central queensland university experience: 1999-2001,' SIGCSE Bull., vol. 34, pp. 89-93, June 2002.

[10] J. Goode, "Increasing diversity in k-12 computer science: Strategies from the field," in ACM SIGCSE Bulletin, vol. 40, pp. 362-366, ACM, 2008.

[11] J. Black, P. Curzon, C. Myketiak, and P. W. McOwan, "A study in engaging female students in computer science using role models," in Proceedings of the 16th annual joint conference on Innovation and technology in computer science education, pp. 63-67, ACM, 2011.

[12] N. Aish, P. Asare, and E. E. Miskioglu, "People like me increasing likelihood of success for underrepresented minorities in stem by providing realistic and relatable role models," in 2017 IEEE Frontiers in Education Conference (FIE), pp. 1-4, IEEE, 2017.

[13] A. Scott, A. Martin, F. McAlear, and S. Koshy, "Broadening participation in computing: examining experiences of girls of color," $A C M$ Inroads, vol. 8, no. 4, pp. 48-52, 2017.

[14] H. Eshan, X. Xu, and M. Cardella, "Representations of underrepresented characters in engineering children books," in Frontiers in Education Conference (FIE), 2016 IEEE, pp. 1-5, IEEE, 2016.

[15] P. Lockwood and Z. Kunda, "Increasing the salience of one's best selves can undermine inspiration by outstanding role models.," Journal of Personality and Social Psychology, vol. 76, no. 2, pp. 214 - 228, 1999.

[16] S. Zirkel, "Is there a place for me? role models and academic identity among white students and students of color," Teachers College Record, vol. 104, no. 2, pp. 357-376, 2002.

[17] A. Sfard and A. Prusak, "Telling Identities: In Search of an Analytic Tool for Investigating Learning as a Culturally Shaped Activity,' Educational Researcher, vol. 34, no. 4, pp. 14-22, 2005.

[18] D. E. Gibson, "Developing the professional self-concept: Role model construals in early, middle, and late career stages," Organization Science, vol. 14, no. 5, pp. 591-610, 2003.

[19] J. MacCallum and S. Beltman, "Role models for young people: What makes an effective role model program," 2002

[20] D. Collins English, "Role model," 2018.

[21] D. Cambridge English, "Role model," 2018.

[22] M. Webster, "Role model," 2018.

[23] P. Bricheno and M. Thornton, "Role model, hero or champion? children's views concerning role models," Educational research, vol. 49, no. 4, pp. 383-396, 2007.

[24] R. Haveman and B. Wolfe, "The determinants of children's attainments: A review of methods and findings," Journal of economic literature, vol. 33, no. 4, pp. 1829-1878, 1995.

[25] V. Grande, A. Berglund, and M. Daniels, "Experiences of teachers in computing as role models: a phenomenographic study," in Proceedings of the 17th Koli Calling Conference on Computing Education Research, pp. 133-137, ACM, 2017.

[26] P. Lockwood, T. C. Marshall, and P. Sadler, "Promoting success or preventing failure: Cultural differences in motivation by positive and negative role models," Personality and Social Psychology Bulletin, vol. 31, no. 3, pp. 379-392, 2005.

[27] P. Lockwood and Z. Kunda, "Superstars and me: Predicting the impact of role models on the self.," Journal of personality and social psychology, vol. 73, no. 1, p. 91, 1997.

[28] K. E. Wohlford, J. E. Lochman, and T. D. Barry, "The relation between chosen role models and the self-esteem of men and women," Sex Roles, vol. 50, no. 7-8, pp. 575-582, 2004.

[29] L. A. Gilbert, "Dimensions of same-gender student-faculty role-model relationships," Sex Roles, vol. 12, no. 1, pp. 111-123, 1985.

[30] J. G. Stout, N. Dasgupta, M. Hunsinger, and M. A. McManus, "Steming the tide: using ingroup experts to inoculate women's selfconcept in science, technology, engineering, and mathematics (stem).," Journal of personality and social psychology, vol. 100, no. 2, p. 255 , 2011.

[31] P. Lockwood, "“someone like me can be successful": Do college students need same-gender role models?," Psychology of Women Quarterly, vol. 30, no. 1, pp. 36-46, 2006.

[32] P. A. Jackson and L. L. Pozzer, "Conceptualizing Identity in Science Education Research: Theoretical and Methodological Issues," in So- ciocultural Studies and Implications for Science Education (C. Milne, K. Tobin, and D. DeGennaro, eds.), vol. 12, ch. 11, pp. 213-230, Springer, 2015

[33] A.-K. Peters, Learning Computing at University: Participation and Identity: A Longitudinal Study. Phd thesis, Uppsala University, Sweden, 2017.

[34] J. Butler, The psychic life of power. Theories in subjection. Stanford University Press, 1997.

[35] V. Burr, Social constructionism. Routledge, 2 ed., 2003.

[36] S. Hall, "Who Needs 'Identity'?," in Questions of cultural identity (S. Hall and P. Du Gay, eds.), SAGE Publications, 1996.

[37] T. Becher and P. Trowler, Academic tribes and territories: Intellectual enquiry and the culture of disciplines. McGraw-Hill Education (UK), 2001.

[38] T. S. Kuhn, The Structure of Scientific Revolutions. The University of Chicago Press, Chicago, USA,3rd edition, 1996.

[39] M. S. Mahoney and T. Haigh, Histories of computing. Harvard University Press, 2011.

[40] B.-L. Gunnarsson, P. Linell, and B. Nordberg, The construction of professional discourse. Routledge, 2014.

[41] J. Meyer and R. Land, Overcoming barriers to student understanding: Threshold concepts and troublesome knowledge. Routledge, 2006.

[42] P. Wegner, "Three computer cultures: Computer technology, computer mathematics, and computer science12," in Advances in computers, vol. 10, pp. 7-78, Elsevier, 1970.

[43] M. Tedre, The science of computing. Shaping a discipline. Taylor \& Francis, 2015

[44] B. C. Smith, On the Origin of Objects. MIT Press, Cambridge, MA, USA, MIT paperback edition, 1998.

[45] D. E. Comer, D. Gries, M. C. Mulder, A. Tucker, A. J. Turner, P. R. Young, and P. J. Denning, "Computing as a discipline," Communications of the ACM, vol. 32, no. 1, pp. 9-23, 1989.

[46] M. Tedre, The science of computing: shaping a discipline. CRC Press, 2014.

[47] S. Nyström, "The dynamics of professional identity formation: Graduates' transitions from higher education to working life," Vocations and Learning, vol. 2, no. 1, pp. 1-18, 2009.

[48] P. Hodkinson, G. Biesta, and D. James, "Understanding Learning Culturally: Overcoming the Dualism Between Social and Individual Views of Learning," Vocations and Learning, vol. 1, pp. 27-47, 2008.

[49] W. Faulkner, "'Nuts and Bolts and People'. Gender-Troubled Engineering Identities," Social Studies of Science, vol. 37, no. 3, pp. 331356, 2007.

[50] B. Rasmussen and T. Håpnes, "Excluding women from the technologies of the future?," Futures, vol. 23, no. 10, pp. 1107-1119, 1991

[51] J. Margolis and A. Fisher, Unlocking the clubhouse. Women in computing. MIT Press, 2002.

[52] M. Goldweber, J. Barr, T. Clear, E. Patitsas, S. Mann, and S. Portnoff, "A Framework for Enhancing the Social Good in Computing Education: A Values Approach Categories and Subject Descriptors," in Proc. final reports on Innovation and technology in computer science education 2012 working groups (ITiCSE-WGR), pp. 16-38, ACM, 2012

[53] E. A. Cech, "Culture of Disengagement in Engineering Education?," Science, Technology, \& Human Values, vol. 39, no. 1, 2014.

[54] C. Laxer, M. Daniels, Å. Cajander, and M. Wollowski, "Evolution of an international collaborative student project," in Proceedings of the Eleventh Australasian Conference on Computing Education-Volume 95, pp. 111-118, Australian Computer Society, Inc., 2009.

[55] D. A. MacKenzie, Mechanizing proof: computing, risk, and trust. MIT Press, 2004

[56] L. MacNell, A. Driscoll, and A. N. Hunt, "What's in a name: Exposing gender bias in student ratings of teaching," Innovative Higher Education, vol. 40, no. 4, pp. 291-303, 2015.

[57] C. A. Moss-Racusin, J. F. Dovidio, V. L. Brescoll, M. J. Graham, and J. Handelsman, "Science faculty's subtle gender biases favor male students," Proceedings of the National Academy of Sciences, vol. 109, no. 41, pp. 16474-16479, 2012.

[58] C. M. Steele, Whistling Vivaldi: How stereotypes affect us and what we can do. Issues of Our Time, W. W. Norton \& Company, 2011.

[59] V. Grande and M. Daniels, "A diversity lens on the last decade of the fie conference: Role models for the engineering community," in Frontiers in Education 2017, 2017. 